\title{
Energy dependence of transverse particle production from SPS to RHIC
}

\author{
András László*, for the NA49 Collaboration \\ RMKI, Budapest \\ E-mail: laszloa@rmki.kfki.hu
}

Transverse momentum spectra up to $4.5 \mathrm{GeV} / \mathrm{c}$ were measured around midrapidity in $\mathrm{Pb}+\mathrm{Pb}$ collisions at $\sqrt{s_{N N}}=17.3 \mathrm{GeV}$, for $\pi^{ \pm}, p, \bar{p}$ and $K^{ \pm}$, by the NA49 experiment. The nuclear modification factors $R_{A A}, R_{A A / p A}$ and $R_{C P}$ were extracted and are compared to RHIC results at $\sqrt{s_{N N}}=200 \mathrm{GeV}$. The modification factor $R_{A A}$ shows a rapid increase with transverse momentum in the covered region. The modification factors $R_{A A / p A}$ and $R_{C P}$ shows a saturation well below unity in the $\pi^{ \pm}$channel. The extracted $R_{C P}$ values follow the $200 \mathrm{GeV}$ RHIC results closely in the available transverse momentum range for all particle species, except for $\pi^{ \pm}$. For $\pi^{ \pm}$ above $2.5 \mathrm{GeV} / \mathrm{c}$ transverse momentum, the measured suppression is smaller than that observed at RHIC. The $K^{+} / \pi^{+}$and $K^{-} / \pi^{-}$ratios were also extracted from the data and are compared to the $200 \mathrm{GeV}$ RHIC results. The values of the former ratio is above, whereas the values of the latter ratio is slightly below the $200 \mathrm{GeV}$ RHIC points.

High-pT Physics at LHC - Tokaj'08

16-19 March 2008

Tokaj, Hungary

* Speaker. 


\section{Introduction}

One of the most interesting features discovered at RHIC is the suppression of particle production at high transverse momenta in central nucleus-nucleus reactions, relative to peripheral ones as well as to $\mathrm{p}+$ nucleus and to $\mathrm{p}+\mathrm{p}$ collisions $[2,3,4,5,6]$. This is generally interpreted as a sign of parton energy loss in hot and dense strongly interacting matter.

The aim of the presented analysis is to investigate the energy dependence of these effects via a systematic study of $\mathrm{Pb}+\mathrm{Pb}$ reactions at top ion-SPS energy, $158 \mathrm{~A} \mathrm{GeV}\left(\sqrt{s_{N N}}=17.3 \mathrm{GeV}\right)$, with the CERN-NA49 detector [7]. A similar study has been published by the CERN-WA98 collaboration for the $\pi^{0}$ channel $[8,9]$. Our analysis extends the existing results to all charged particle channels, i.e. $\pi^{ \pm}, p, \bar{p}$ and $K^{ \pm}$.

Invariant yields were extracted as a function of transverse momentum $p_{T}$ in the range from 0.3 to $4.5 \mathrm{GeV} / \mathrm{c}$ in the rapidity interval $-0.3 \leq y \leq 0.7$ (midrapidity), at different collision centralities $[1,10]$. Identification of particle types is crucial, because the particle composition of hadron spectra changes rapidly with transverse momentum and differs significantly from that observed at RHIC energies.

Using the identified single particle spectra from [1] and the charged pion spectra from [11, 12, 13], the nuclear modification factors were calculated. These are defined as

$$
\begin{aligned}
& R_{A_{1}+A_{2} / A_{3}+A_{4}}^{B C}=\frac{\left\langle N_{B C}\left(A_{3}+A_{4}\right)\right\rangle}{\left\langle N_{B C}\left(A_{1}+A_{2}\right)\right\rangle} \cdot \frac{\operatorname{yield}\left(A_{1}+A_{2}\right)}{\operatorname{yield}\left(A_{3}+A_{4}\right)}, \\
& R_{A_{1}+A_{2} / A_{3}+A_{4}}^{W}=\frac{\left\langle N_{W}\left(A_{3}+A_{4}\right)\right\rangle}{\left\langle N_{W}\left(A_{1}+A_{2}\right)\right\rangle} \cdot \frac{\operatorname{yield}\left(A_{1}+A_{2}\right)}{\operatorname{yield}\left(A_{3}+A_{4}\right)},
\end{aligned}
$$

where $\left\langle N_{B C}\right\rangle$ and $\left\langle N_{W}\right\rangle$ are, respectively, the average number of: binary collisions and wounded nucleons, calculated for the reactions $A_{1}+A_{2}$ and $A_{3}+A_{4}[14,15] . R_{A A}, R_{p A}$ and $R_{C P}$ are used to denote the special cases $R_{A+A / p+p}, R_{p+A / p+p}$ and $R_{\text {Central/Peripheral }}$ for $A+A$ reactions, whereas $R_{A A / p A}$ abbreviates $R_{A+A / p+A}$. These were calculated and compared with the $\sqrt{s_{N N}}=200 \mathrm{GeV}$ RHIC results [3, 5].

Also the $K^{+} / \pi^{+}$and $K^{-} / \pi^{-}$ratios were extracted from the data and are compared to $\sqrt{S_{N N}}=$ $200 \mathrm{GeV}$ RHIC results of [3].

\section{Analysis details}

The centrality of events was determined using the energy of projectile spectators deposited in a downstream Veto Calorimeter (VCAL). Careful study of the detector response and Glauber calculations were performed in order to obtain $\left\langle N_{B C}\right\rangle$ and $\left\langle N_{W}\right\rangle$ as a function of centrality $[1,14,15]$.

Due to the rapid decrease of the $p_{T}$ spectra, special care was taken to achieve good signal-tonoise ratio in the high- $p_{T}$ region. Possible background tracks were rejected with a twofold filtering procedure: (1) discontinuous tracks were discarded, and (2) tracks originating from the acceptance border were rejected $[1,10]$.

Particles were identified at the spectrum level, using specific ionization $\left(\frac{d E}{d x}\right)$ fits $[1,10]$. 
The resulting particle spectra were corrected for feed-down (5 to 30\%), decay loss (20\% to $0 \%$ ), tracking inefficiency (below 10\%), non-target contribution (below 5\%), and geometric acceptance. The fake-rate, momentum smearing, and momentum scale uncertainty proved to be negligible. The correction details are discussed in $[1,10]$.

After full correction, the systematic errors are about $2.2 \%$ for $\pi^{+}, \pi^{-}$and $K^{-}, 3.7 \%$ for $p$, $4.5 \%$ for $K^{+}$, and $6.5 \%$ for $\bar{p}[1,10]$.

\section{Results and discussion}

The inclusive particle spectra of $[1,11,12,13]$ allow to calculate the nuclear modification factors $R_{A A}, R_{p A}, R_{A A / p A}$ and $R_{C P}$ for top ion-SPS energy. Additionally, a close-by energy data set [16] was considered for the $\mathrm{p}+\mathrm{W} / \mathrm{p}+\mathrm{p}$ yield ratios. These results are compared to similar quantities at the top RHIC energy [3, 5]. All of this is shown in Fig. 1, along with pQCD-based energy loss model predictions for $R_{C P}$ [17].

It is seen that the $R_{A A}$ and the $R_{p A}$ at the top ion-SPS energy increase monotonically with $p_{T}$ in the covered region. This is sometimes referred to as the Cronin effect. A widely accepted explanation for this phenomenon is initial multiple scattering on either partonic or hadronic level depending on the valid particle production picture. It is also observed that the $R_{A A}^{B C}$ points stay below $R_{p A}^{B C}$, whereas the $R_{A A}^{W}$ points are above $R_{p A}^{W}$ both at top ion-SPS energy and at RHIC. The $R^{W}$ modification factors start approximately from unity.

If the multiple scattering interpretation of the Cronin effect is valid, it is natural to expect it to contribute much less to the nuclear modification factors $R_{A A / p A}$ and $R_{C P}$. Therefore, if looking for nuclear effects other than multiple scattering, these ratios should also be considered. The $R_{A A / p A}^{B C}$ and $R_{C P}^{B C}$ stay well below unity at top ion-SPS energy, but show much less suppression than at the top RHIC energy in the $\pi^{ \pm}$channel above $p_{T}>2 \mathrm{GeV} / \mathrm{c}$. For other particle channels, the behavior of the $R_{C P}^{B C}$ points at SPS and at RHIC seem to be rather similar, except for $\bar{p}$, which may be affected by the larger systematic errors due to the antiproton detection technique at the experiment NA49. The pQCD-based energy loss model seems to give a fair description of the $R_{C P}$ data points at SPS energy.

To further test the predictions of the pQCD-based model, other particle ratios may also be looked at. It is found that the $\bar{p} / \pi^{-}$data are not reproduced, as shown in Fig. 2.

To get information about the strangeness production differentiated in centrality and $p_{T}$, the $K^{+} / \pi^{+}$and $K^{-} / \pi^{-}$ratios were also extracted from the data, and are compared to $\sqrt{s_{N N}}=200 \mathrm{GeV}$ data [3]. All this is shown in Fig. 3. A monotonic increase of both ratios with centrality and $p_{T}$ is observed. The $K^{-} / \pi^{-}$points at top ion-SPS energy are slightly below the top RHIC energy points, whereas the $K^{+} / \pi^{+}$points at top ion-SPS energy are above the RHIC points.

\section{Summary}

The measured $R_{A A}$ and $R_{p A}$ data show a monotonic increase in the covered $p_{T}$ region at SPS energy. The $R_{A A}^{B C}$ points stay below $R_{p A}^{B C}$. The $R_{A A / p A}^{B C}$ and $R_{C P}^{B C}$ stay well below unity at SPS energy for $\pi^{ \pm}$, but much less suppression is observed than at RHIC above $p_{T}>2 \mathrm{GeV} / \mathrm{c}$.

The $R^{W}$ data start from one and $R_{A A}^{W}$ is above $R_{p A}^{W}$ both at SPS and at RHIC energy. 

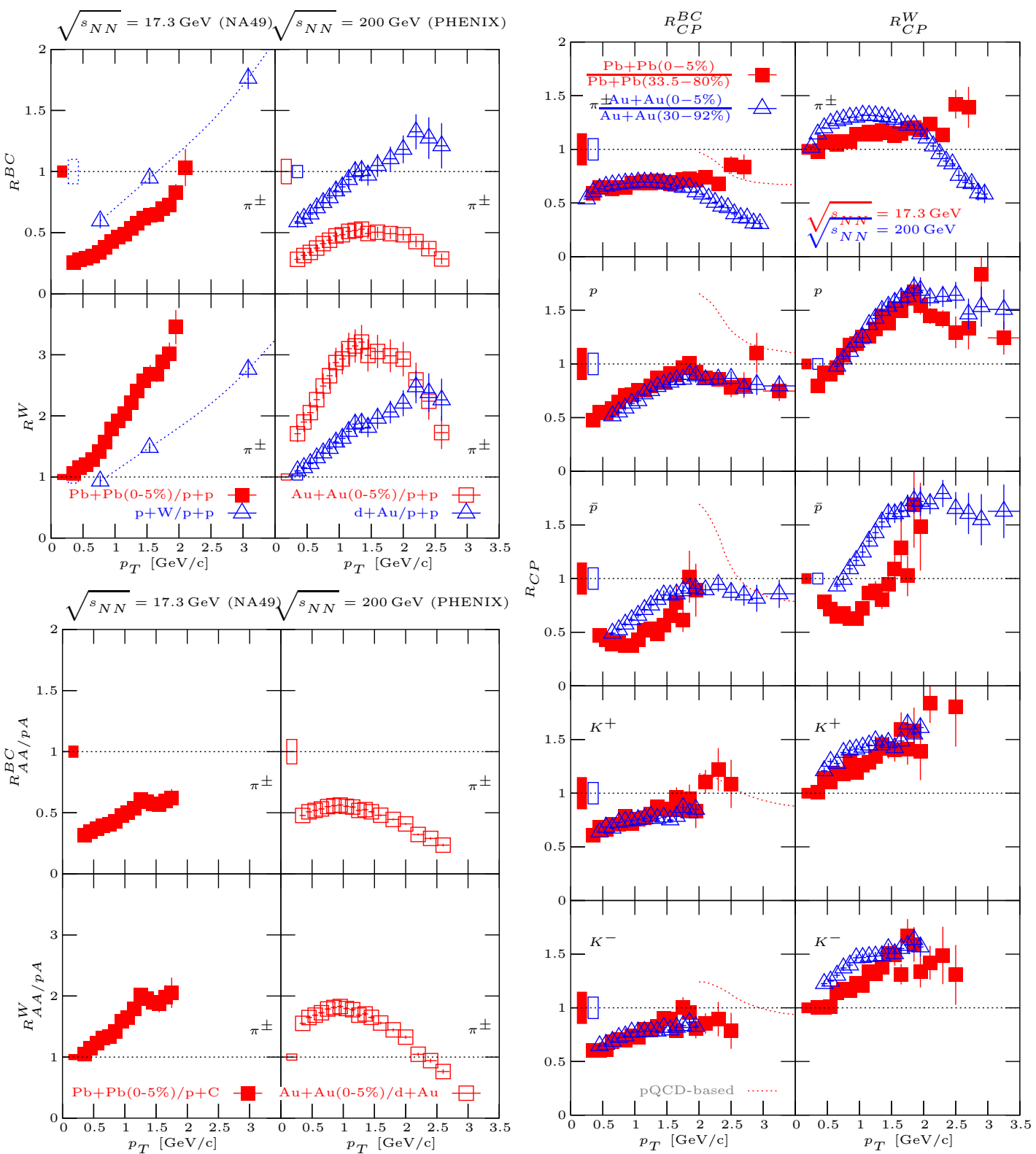

Figure 1: $R_{A A}$ (top left), $R_{A A / p A}$ (bottom left), and $R_{C P}$ (right) nuclear modification factors, measured at $\sqrt{s_{N N}}=17.3 \mathrm{GeV}[1,11,12,13]$, and compared to $\sqrt{s_{N N}}=200 \mathrm{GeV}$ data [3, 5]. To supplement the existing SPS energy data, a $\sqrt{s_{N N}}=19.4 \mathrm{GeV} \mathrm{p}+\mathrm{W} / \mathrm{p}+\mathrm{p}$ data set was also used [16]. The dotted lines in the top left panel are drawn to guide the eye. The dotted lines in the right panel indicate a pQCD-based energy loss model prediction [17].

The $R_{C P}^{B C}$ data follow the RHIC points closely, except for $\bar{p}$ particles and for $\pi^{ \pm}$at $p_{T}>$ $2 \mathrm{GeV} / \mathrm{c}$.

The $R_{C P}^{B C}$ points are explained by pQCD-based energy loss calculation as in [17], however the produced-baryon/meson ratios are not reproduced. Possibly pQCD is not applicable in this kinematic region.

The $K^{+} / \pi^{+}$and $K^{-} / \pi^{-}$ratios show a monotonic increase in centrality and $p_{T}$ both at SPS 


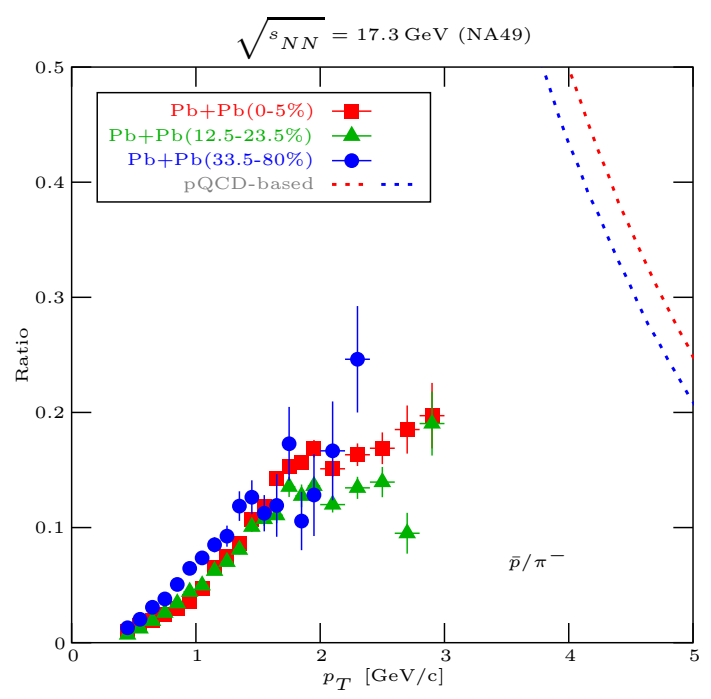

Figure 2: Produced-baryon to meason ratios in $\mathrm{Pb}+\mathrm{Pb}$ collisions, at $\sqrt{s_{N N}}=17.3 \mathrm{GeV}$ [1]. The dotted lines indicate a pQCD-based energy loss model prediction [17].
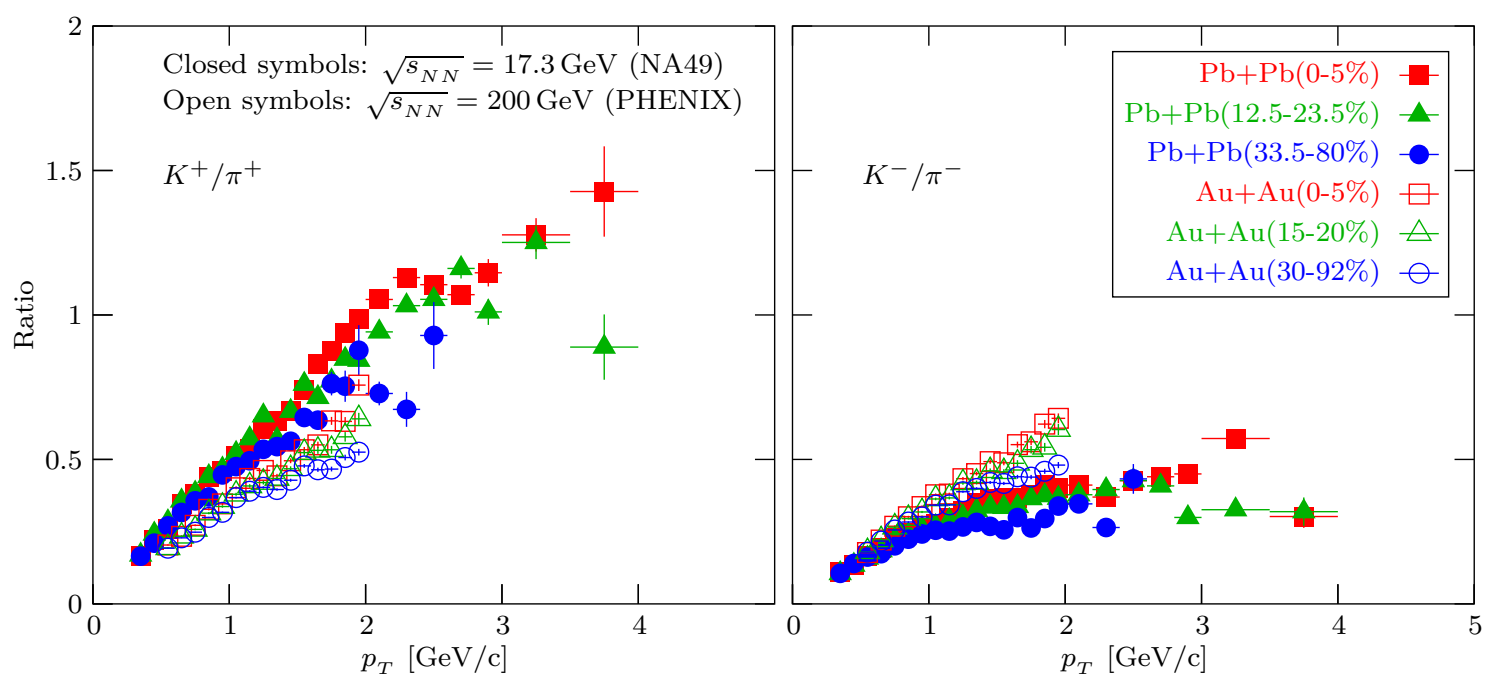

Figure 3: $K^{+} / \pi^{+}$and $K^{-} / \pi^{-}$ratios in $\mathrm{Pb}+\mathrm{Pb}$ collisions, at $\sqrt{s_{N N}}=17.3 \mathrm{GeV}$ [1] and at $\sqrt{s_{N N}}=200 \mathrm{GeV}$ [3].

and RHIC energy. The $K^{-} / \pi^{-}$points are slightly below the RHIC points, whereas the $K^{+} / \pi^{+}$ points are above the points measured at RHIC energy.

\section{Acknowledgments}

This work was supported by the US Department of Energy Grant DE-FG03-97ER41020/A000, the Bundesministerium fur Bildung und Forschung, Germany, the Virtual Institute VI-146 of Helmholtz 
Gemeinschaft, Germany, the Polish Ministry of Science and Higher Education (1 P03B 006 30, 1 P03B 097 29, 1 P03B 121 29, 1 P03B 127 30), the Hungarian Scientific Research Fund (OTKA 68506), the Korea Research Foundation (KRF-2007-313-C00175), the Bulgarian National Science Fund (Ph-09/05), the Croatian Ministry of Science, Education and Sport (Project 098-09828872878) and Stichting FOM, the Netherlands.

\section{References}

[1] C. Alt et al. (the NA49 Collaboration), Phys. Rev. C77 (2008) 034906.

[2] S. S. Adler et al. (the PHENIX Collaboration), Phys. Rev. C69 (2004) 034909.

[3] S. S. Adler et al. (the PHENIX Collaboration), Phys. Rev. C69 (2004) 034910.

[4] S. S. Adler et al. (the PHENIX Collaboration), Phys. Rev. Lett. 91 (2003) 072303.

[5] S. S. Adler et al. (the PHENIX Collaboration), Phys. Rev. C74 (2006) 024904.

[6] L. Ruan (the STAR Collaboration), J. Phys. G31 (2005) s1029.

[7] S. Afanasiev et al. (the NA49 Collaboration), Nucl. Instr. Meth. A430 (1999) 210.

[8] M. M. Aggarwal et al. (the WA98 Collaboration), Eur. Phys. J. C23 (2002) 225.

[9] M. M. Aggarwal et al. (the WA98 Collaboration), Phys. Rev. Lett. 100 (2008) 242301.

[10] A. László, NA49 Technical Note (2007) [EDMS : 879787].

[11] C. Alt et al. (the NA49 Collaboration), Eur. Phys. J. C45 (2006) 343.

[12] C. Alt et al. (the NA49 Collaboration), Eur. Phys. J. C49 (2007) 897.

[13] C. Alt et al. (the NA49 Collaboration), Eur. Phys. J. C49 (2007) 919.

[14] A. László, NA49 Technical Note (2006) [EDMS : 815907 ].

[15] A. László, NA49 Technical Note (2007) [EDMS : 885329].

[16] D. Antreasyan et al., Phys. Rev. D19 (1979) 764.

[17] X. N. Wang, Phys. Lett. $\mathbf{B 5 9 5}$ (2004) 165. 